\title{
Liquid Crystalline Properties of the Copolyesters Prepared from Resorcinol, p-Hydroxybenzoic Acid, and Terephthalic Acid
}

\author{
Jung-Il JIN, ${ }^{*}$ Suck-Hyun LeE, ${ }^{* *}$ Ho-Jin PARK, ${ }^{* * *}$ \\ and Il-Joong $\mathrm{KIM}^{*}$ \\ *Chemistry Department, College of Sciences, Korea University, \\ 1-Anam Dong, Seoul 136-701, Korea \\ ** Chemical Engineering Department, A-Joo University, \\ Suwon City 440-749, Korea \\ ${ }^{* * *} R \&$ D Center, KOLON Inc., Gumi City 730-030, Korea
}

(Received January 11, 1989)

\begin{abstract}
A series of aromatic copolyesters was prepared in melts from 1,3-diacetoxybenzene, terephthalic acid and $p$-acetoxybenzoic acid, and the copolyesters' liquid crystalline and other properties were investigated. The compositions containing more than $65 \mathrm{~mol} \%$ the $p$-oxybenzoyl (POB) unit exhibited thermotropic behavior, whereas those with less than $40 \mathrm{~mol} \%$ the POB structure were not thermotropic. The copolyesters with an intermediate level $(50-60 \mathrm{~mol} \%)$ of POB units showed an interesting, shear flow induced formation of mesophases. The possibility of compositional heterogeneity in the copolyesters was demonstrated by collecting and characterizing insoluble fractions.
\end{abstract}

KEY WORDS Aromatic Copolyesters / Resorcinol / Terephthalic Acid / pHydroxybenzoic Acid / Liquid Crystalline / Compositional Heterogeneity / Shear Induction

The structure-property relationships of thermotropic aromatic copolyesters are of commercial and academic interest. A few thermotropic aromatic copolyesters of commercial importance are presently available on the market, and they are known to have many distinctive properties such as high modulus, very low mold shrinkage, low melt viscosity, etc. It has been rather well established, both theoretically and experimentally, that a rigid rodlike structure is a prerequisite for a polymer to be liquid crystalline. ${ }^{1-3}$ Two representative rigid rodlike polyesters, poly( $p$-oxybenzoate) and poly( $p$-phenyleneterephthalate) are expected to be thermotropic. They, however, have to be structurally modified to make melt processable. Inclusion of unsymmetric substituents on the phenyl rings, modification to include flexible spacers or kinky units in the main chain, and copolymerizations are the most frequently employed approaches to structurally modify these two polyesters, lowering their melting points and rendering them melting processable. $^{4.5}$ There are quite a few articles by us, ${ }^{4-11}$ and others, ${ }^{3,12-19}$ that describe how those modifications change the liquid crystalline and other properties of the resulting polyesters.

In this study we prepared a series of copolyesters consisting of terephthalic acid (TPA), p-hydroxybenzoic acid (POB), and resorcinol (RES) units. The last moiety was included to create a kinky structure. The copolyesters were prepared by melt transesterification polymerization from terephthalic acid (TPA), 1,3-diacetoxybenzene (RD), and $p$ acetoxybenzoic acid (ABA).

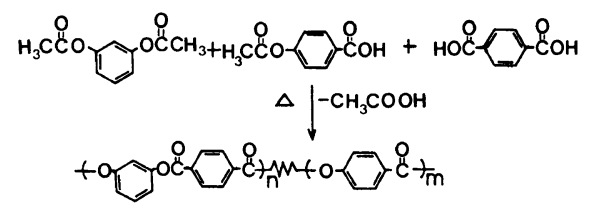


The $\mathrm{mol}^{\%} \%$ of the POB moiety, $m$, in the copolyesters was varied: $0 \%, 16 \%, 31 \%, 53 \%$, $63 \%, 70 \%, 79 \%$, and $86 \%$. The polymers' thermal, rheological and liquid crystalline properties were studied. Each copolyester is named mR-RES X; $m$ denotes melt polymerization, $\mathrm{R}$ random copolyester, RES resorcinol as a bent monomer and $\mathrm{X} \mathrm{mol} \%$ of POB unit contained in the copolyester. For example, mR-RES 70 contains $70 \mathrm{~mol}_{\%}^{\%}$ of p-oxybenzoyl structure in the chain. A preliminary report was presented earlier. ${ }^{20}$

\section{EXPERIMENTAL}

\section{Materials}

$p$-Hydroxybenzoic acid, resorcinol, and terephthalic acid used were of reagent grade. $p$ Hydroxybenzoic acid and resorcinol were acetylated in excess boiling acetic anhydride solutions for $4 \mathrm{~h}^{21}$ and were purified by recrystallization from acetic acid and by vacuum distillation. The purity was checked by TLC, NMR, and IR spectroscopy. Other chemicals were of reagent grade and used without further purification.

\section{Polymerization}

The copolyesters were prepared in melts from RD, TPA, and ABA. The composition of the copolyesters was varied by changing the mol fraction of ABA. The molar ratio of $\mathrm{RD}$ to TPA was kept constant at unity. A typical polymerization method used for the preparation of mR-RES 70 is as follows: In a 2 gallon autoclave equipped with a condenser, an agitator, a nitrogen purge line and torque $(\mathrm{kW})$ measuring and recording devices, $215.52 \mathrm{~g}$ $(1.11 \mathrm{~mol})$ of resorcinol diacetate, $339.93 \mathrm{~g}$ $(2.22 \mathrm{~mol})$ of $p$-acetoxybenzoic acid, $184.55 \mathrm{~g}$ $(1.11 \mathrm{~mol})$ of terephthalic acid and $30 \mathrm{ml}$ of acetic anhydride were charged. The reactor was purged with dry nitrogen to expel oxygen.

The polymerization was conducted in two stages. In the first stage of reaction with nitrogen purging, the temperature of the mixture was raised to $200^{\circ} \mathrm{C}$ over an hour and then it was further raised to $240^{\circ} \mathrm{C}$ in a $50 \mathrm{~min}$ with continued nitrogen purging. The temperature of the reaction mixture was maintained at $240^{\circ} \mathrm{C}$ until most of the acetic acid was evolved. Throughout this operation stirring was maintained at $200 \mathrm{rpm}$. In the second stage, a vacuum was applied, reducing the pressure gradually from one atmosphere to 0.1 Torr while the temperature of the mixture was raised from $240^{\circ} \mathrm{C}$ to $295^{\circ} \mathrm{C}$. The stirring rate was reduced to $50 \mathrm{rpm}$. The extent of the reaction was followed by observing readings on the torque meter (Figure 1). At the end of the polymerization, the polymer formed was extruded into water and cut into chips. A large scale polymerization $(40 \mathrm{~kg} /$ Batch $)$ also was conducted, following the same procedure, for the preparation of $\mathrm{mR}$-RES 70 .

\section{Characterization}

Melting points of the monomers and intermediates were measured on a DSC (PerkinElmer, DSC-2) or on a Fisher-Johns melting point apparatus. Elemental analysis was performed by the Analytical Service Department of the Korea Research Institute of Chemical Technology. To determine the compositions of the copolyesters, the polymers were hydrolyzed by combining about $50 \mathrm{mg}$ of polymer with $6 \mathrm{~g}$ of $\mathrm{KOH}$ and $50 \mathrm{ml}$ of ethanol in a $250 \mathrm{ml}$ round bottom flask. The mixture was refluxed for $30 \mathrm{~h}$ with stirring. The hydrolyzed products were acidified with $12 \mathrm{M} \mathrm{HCl}$ to a $\mathrm{pH}$ of 4 . The mixture was evaporated to dryness and the residue extracted 3 times with $20 \mathrm{ml}$ of methanol. The total volume was adjusted to $100 \mathrm{ml}$. The sample was analyzed by high pressure liquid chromatography using a Water's Associate Model-244 HPLC equipped with a UV detector ( $254 \mathrm{~nm}$ ) and a Lichrosorb RP-18 $10^{1}+10^{2}+10^{3}$ column. As an elution solvent, a water-methanol ( $7: 3$ by volume) mixture acidified with $1 \%$ acetic acid was used. The volume of sample solution injected was $5 \mu \mathrm{l}$ and the flow rate of eluent solvent was 1.0 
$\mathrm{ml} \mathrm{min}{ }^{-1}$.

Solution viscosities of the polymers were measured at $30^{\circ} \mathrm{C}$ by a Cannon-Ubbelohde viscometer using a $p$-chlorophenol-2,3,4,5,6pentaluorophenol-chloroform $(3: 3: 4$ by volume) mixture. The concentration of the solution was $0.1 \mathrm{~g} / 100 \mathrm{ml}$.

$\bar{M}_{n}$ and $\bar{M}_{n}$. were measured on a Water's GPC Model-I equipped with a Styragel $10^{1}+10^{2}+10^{3}$ column and differential refractometer detector using polystyrene as a standard. The polymers were dissolved in $o$ chlorophenol-chloroform ( $1: 4$ by volume) at a concentration of $0.25 \mathrm{wt} \%$ and were eluted at room temperature by the same solvent at a rate of $1.0 \mathrm{ml} \mathrm{min}^{-1}$.

Microscopic observations were made on a Leitz Ortholux polarizing microscope equipped with a Mettler FP-5 hot stage. Thermal analyses were performed on a DSC-2 (PerkinElmer) instrument at a heating rate of $10 \mathrm{C} \mathrm{min}^{-1}$. The polymers' thermal stability was studied under nitrogen atmosphere on a TGA (Perkin-Elmer TGS-2) at a heating rate of $20^{\circ} \mathrm{Cmin}^{-1}$. Crystalline properties were examined by X-ray diffractometry (D-Max IIIB, Rigaku) using a Ni-filtered $\mathrm{Cu}-K_{\alpha}$ source.

The homogeneity of selected compositions was studied by dissolving the polymers in $o$ chlorophenol at $3 \mathrm{wt} \%$ concentration. These solutions were examined on a polarizing light microscope with polars crossed. The undissolved particles were separated by a high speed (3000 rpm) centrifuge and analyzed by NMR spectroscopy, X-ray diffractometry and monomer composition analysis.

The viscoelastic properties were studied on a rheometer, RDS-7700 (Rheometrics Co.).

\section{RESULTS AND DISCUSSION}

Melt Viscosity Change During Polymerization

According to Figure 1, the torque encountered by the reactor stirrer in the polymerization of the LC forming composition, an indicator of melt viscosity, initially increases

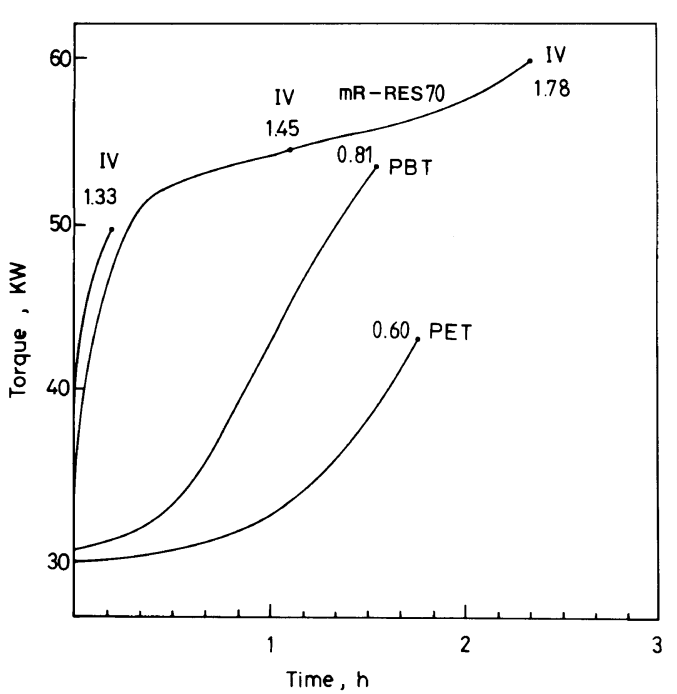

Figure 1. Torque of the agitator in the polymerization reactor $v s$. polymerization time. The numbers indicate the inherent viscosity values at the points indicated. ABA content, $70 \mathrm{~mol}^{\%}$; Batch size, $40 \mathrm{~kg}$ batch $^{-1}$; PET, poly(ethylene terephthalate); PBT, poly(butylene terephthalate).

rapidly, but the rate of increase abruptly diminishes after a certain period of polymerization time. The initial rate of viscosity increase is much higher than those observed for isotropic polyesters such as poly(ethylene terephthalate) and poly(butylene terephthalate). The isotropic compositions, however, exhibited a continued exponential increase in melt viscosity with reaction time. We believe that such a difference arises from the relatively rigid nature of LC compositions. Initially, the increase in molecular weight or in chain length of rigid molecules causes a sharp increase in melt viscosity when compared to flexible, isotropic polymers. Then, as the length of the rigid chains continues to grow and reaches a threshold value, the polymer becomes able to form a homogeneous nematic phase. As is well known, the melt viscosity of a nematic state is very shear sensitive and susceptible to shear-thinning. This should result in a diminished rate of increase in the melt viscosity or torque experienced by the stirrer in the 
Table I. Properties of copolyesters

\begin{tabular}{|c|c|c|c|c|c|c|c|c|}
\hline \multirow{2}{*}{ Polymer } & \multicolumn{2}{|c|}{$\mathrm{ABA} / \mathrm{mol}^{\%} / \mathrm{o}$} & \multirow{2}{*}{$\frac{\eta_{\mathrm{inh}}}{\mathrm{dl} \mathrm{g}^{-1}}$} & \multirow{2}{*}{$\frac{T_{\mathrm{g}}}{\mathrm{C}}$} & \multirow{2}{*}{$\frac{T_{\mathrm{m}}}{{ }^{\circ} \mathrm{C}}$} & \multirow{2}{*}{ L.C. } & \multirow{2}{*}{$\bar{M}_{w}$} & \multirow{2}{*}{$\bar{M}_{w} / \bar{M}_{n}$} \\
\hline & Feed & Actual & & & & & & \\
\hline mR-RES 0 & 0.0 & 0.0 & 1.00 & 157 & 297 & No & 35,000 & 5.7 \\
\hline mR-RES 16 & 18.2 & 16.0 & 0.76 & 157 & 289 & No & \multicolumn{2}{|c|}{ Not measured } \\
\hline mR-RES 31 & 33.3 & 31.0 & 0.76 & 144 & 269 & No & 31,000 & 6.1 \\
\hline mR-RES 53 & 50.0 & 53.4 & 0.68 & 142 & - & By shear & 39,000 & 6.7 \\
\hline mR-RES 63 & 60.1 & 63.1 & 0.71 & 142 & - & By shear & \multicolumn{2}{|c|}{ Not measured } \\
\hline mR-RES 70 & 66.7 & 69.6 & 1.21 & 127 & - & Yes & 57,000 & 6.5 \\
\hline mR-RES 79 & 75.0 & 78.9 & 1.68 & 120 & - & Yes & \multicolumn{2}{|c|}{ Insoluble } \\
\hline mR-RES 86 & 82.4 & 85.5 & 1.55 & 161 & - & Yes & \multicolumn{2}{|c|}{ Insoluble } \\
\hline mR-RES 100 & 100 & - & 0.45 & 329 & - & - & \multicolumn{2}{|c|}{ Insoluble } \\
\hline
\end{tabular}

latter part of polymerization. On the other hand, the melt viscosity of isotropic polymers is very well known to increase with molecular weight and is much less sensitive on shear. This point will be discussed later.

\section{General Properties, Glass and Melting Tran- sitions and Liquid Crystallinity}

The compositions, inherent viscosities, thermal transition temperatures, liquid crystallinity and molecular weights of the polymers are listed in Table I. According to the composition analysis results given in Table $\mathrm{I}$, actual copolymer compositions are fairly close to those of the feed. In general, with the exception of the first two copolymers, mR-RES 16 and mR-RES 21, the copolyesters contain slightly greater levels of $p$-oxybenzoyl (POB) unit than the feeds. Probably a greater loss of terephthalic acid and 1,3-diacetoxybenzene during polymerization causes this. Elemental analyses, however, did not provide firm conclusion for the copolymer compositions, due to the virtual independence of $\mathrm{C}$ and $\mathrm{H}$ content on composition.

The value of the solution viscosities measured in a $p$-chlorophenol-2,3,4,5,6-pentalfuorophenol-chloroform ( $3: 3: 4$ by volume) solution ranges from about 0.5 to 1.7 . The $\bar{M}_{w}$ values ranged from 31000 to 57000 . The polydispersity indices were about 6-6.5. Glass transition temperature slowly dimin- ished with POB content and showed minimum at $79 \mathrm{~mol}_{0}^{\circ}$. Afterward, it increased sharply.

The copolyesters with less than $70 \mathrm{~mol} \%$ POB formed isotropic phases upon melting, judging by the textures of polymer melts observed through a polarizing microscope. The copolyesters containing 53 and $63 \mathrm{~mol} \%$ POB, i.e., mR-RES 53 and mR-RES 63, however, were observed to form nematic textures under high shear. The compositions containing up to $31 \mathrm{~mol} \%$ of the POB unit were crystalline and exhibited melting endotherms on DSC thermograms. The DSC melting peaks of the copolyesters containing 53 to $70 \mathrm{~mol} \%$ POB, however, were very small even after prolonged heat-treatment (at 200 or 220 or $240^{\circ} \mathrm{C}$ ). This implies that these copolyesters are essentially amorphous in nature. X-Ray diffraction studies of the copolyesters further supported these DSC observations. Compositions with POB content higher than $79 \mathrm{~mol} \%$ are crystalline after annealing at $200^{\circ} \mathrm{C}$ for $15 \mathrm{~h}$, but their melting transition temperatures could not be observed on a DSC due to their thermal decomposition before reaching $T_{\mathrm{m}}$. Figure 2 shows a typical amorphous $\mathrm{X}$-ray diffraction pattern of the mR-RES 70 copolyester.

Figure 3 shows an optical texture observed for the shear-induced liquid crystalline phase for mR-RES 53. This picture was taken at $280^{\circ} \mathrm{C}$ after shearing the polymer melt between a glass slide and cover slip. This texture grad- 


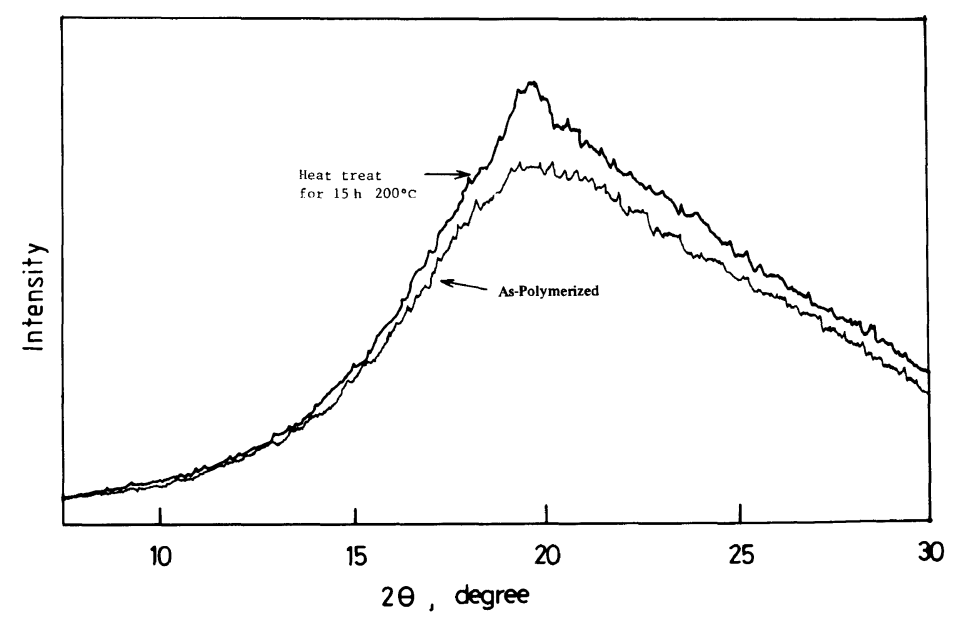

Figure 2. X-Ray diffractogram of $m R$-RES 70: X-ray source; $\mathrm{Cu}-K_{\alpha} \mathrm{Ni-filtered} \mathrm{radiation;} \mathrm{slit,} \mathrm{DS} 1^{\circ}$, RS $0.15^{\circ}$, S.S. $1^{\circ}$; voltage, current, $35 \mathrm{kV}, 15 \mathrm{~mA}$; scan speed, $1^{\circ} \mathrm{C} \mathrm{min}^{-1}$.

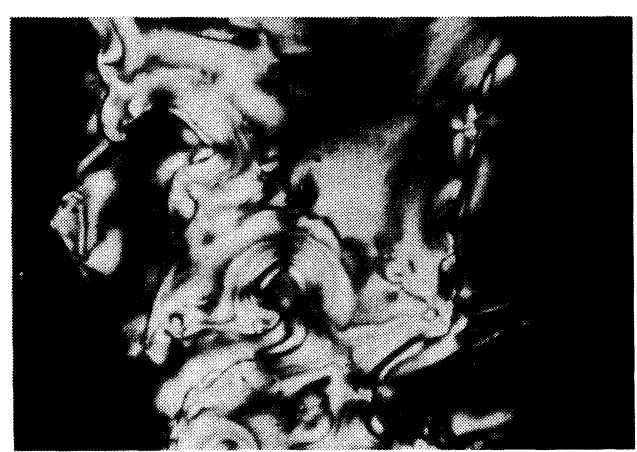

Figure 3. Photomicrograph of shear-induced liquid crystalline phase of mR-RES 53 taken on a cross polarizing microscope at $280^{\circ} \mathrm{C}$ (magnification $80 \times$ ).

ually disappeared when shearing was stopped and disappeared completely in $15 \mathrm{~min}$. This clearly indicates the shear induced formation of the anisotropic phase. A similar phenomenon was observed for mR-RES 63. $\mathrm{mR}$ RES 70 and those containing higher levels of ABA structure were able to form nematic phases in quiescent melts (Figure 4).

\section{Analysis of the Insoluble Copolymers}

When mR-RES 70 was dissolved in $o$ chlorophenol, insoluble, swollen particles were observed. The amount of insolubles was $4.6 \%$ by weight. The insoluble particles, collected by

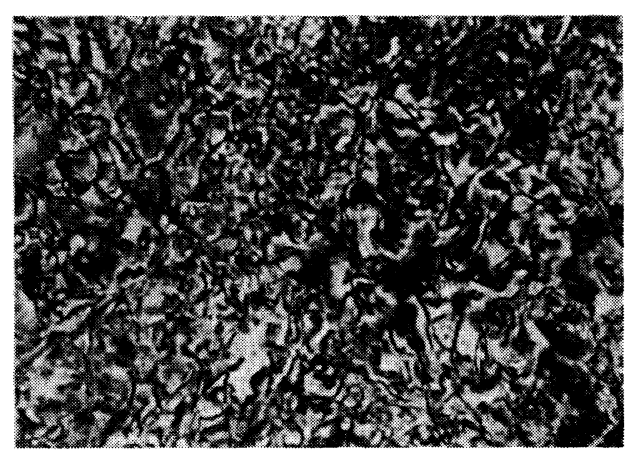

Figure 4. Photomicrograph taken at $290^{\circ} \mathrm{C}$ of the optical texture observed for the melt of mR-RES $70(80 \times)$.

centrifugation from the solution, were found to be highly crystalline (Figure 5), while the bulk polymer was nearly amorphous (Figure 2). The insoluble particles contained a higher level of POB unit $(88.5 \mathrm{~mol} \%)$ than the bulk polymer $(69.6 \mathrm{~mol} \%)$. Moreover, the X-ray diffraction pattern of the insolubles was very similar to that of poly ( $p$-oxybenzoate) (PPOB), but with an additional peak at a $2 \theta$ of $16^{\circ}$ (Figure 5).

The melting point of the insoluble portion determined by DSC was $298^{\circ} \mathrm{C}$. This demonstrates that even the insoluble polymer portion is not a homopolymer of p-hydroxybenzoic acid. The insoluble polymers probably 


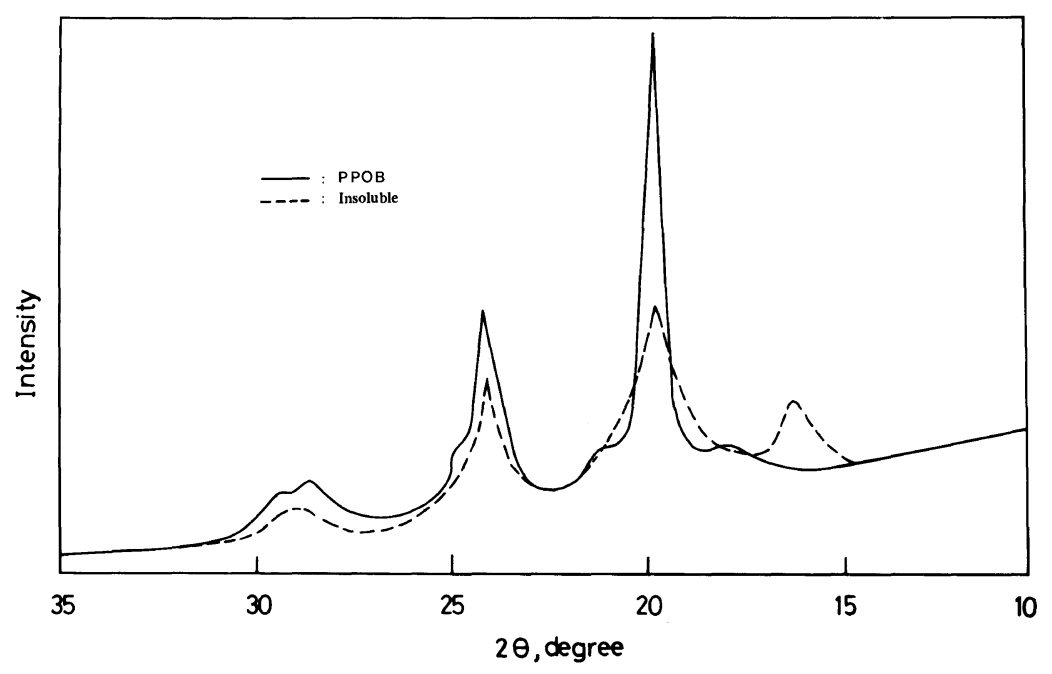

Figure 5. X-Ray diffractogram of insoluble particles collected from mR-RES 70 .

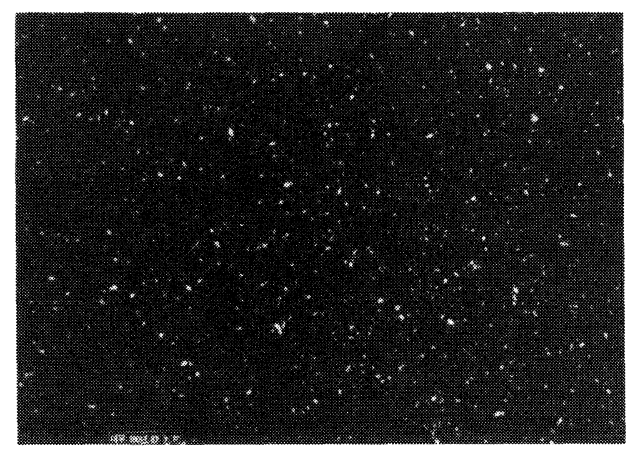

Figure 6. Photomicrograph of the $O$-chlorophenol solution of mR-RES 70 taken with crossed polars. Concentration of the solution was $3 \%$ (magnification $80 \times)$.

consist primarily of short blocks of POB units along the chains. We believe that there is no possibility of the formation of poly( $p$-oxybenzoate) homopolymer or copolymers containing long blocks of poly( $p$-oxybenzoate) structure in light of the fact that this copolymer is completely soluble in a mixed solvent of an $o$-chlorophenol/chloroform while poly ( $p$-oxybenzoate) is not. A $3 \%$ solution of mR-RES 70 in $o$-chlorophenol was examined on a polarizing microscope. Figure 6 shows an optical photomicrograph obtained from the $o$ chlorophenol solution. Small, individual and segregated, birefringent particles are evident. These observations further support our contention that this copolyester is not truly homogeneous in composition.

Baird et $a .^{22}$ studied the injection molded specimen of POB/PET copolyester and found that the skin part contained a higher amount of the POB moiety than the core. They attributed this result to heterogeneous comonomer sequences in the polymer backbone. Gilkey and Caldwell ${ }^{23}$ also reported that, when the copolyesters of meta- and para-hydroxybenzoic acids were dissolved in tetrachloroethane, they found that the copolyester with paracontent lower than $30 \mathrm{~mol} \%$ dissolved completely, but that insoluble particles existing for the compositions with higher levels of the para isomer. They reported that the insoluble particles consisted of blocks richer in POB. Our observations, together with others, imply that, when the reactivities of the comonomers are not equal, compositional heterogeneity may result from copolymerizations. In short, the copolymer with a very high content of POB unit may not be truly random in monomer sequence. 


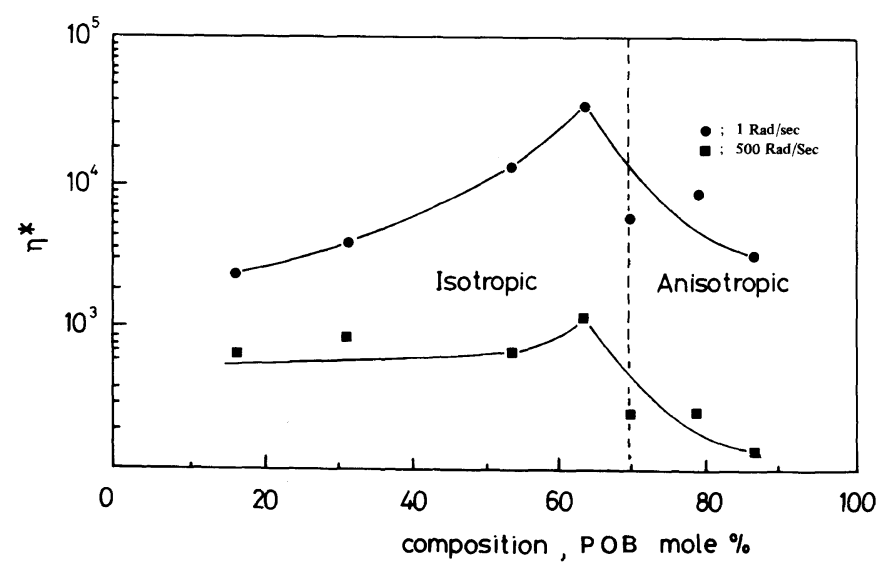

Figure 7. Dependence of complex viscosity on the composition of copolyesters. Temp, $295^{\circ} \mathrm{C}$; strain, $15 \%$; gap between disc. \& plate, $0.5 \mathrm{~mm}$; apparatus, RDS-7700.

\section{Rheological Properties ${ }^{24-30}$}

The complex viscosities of the copolyesters are presented in Figure 7. Melt viscosities of the polymers slowly increase with the content of POB and then abruptly drop at POB content higher than $63 \mathrm{~mol} \%$. The viscosity descending point coincides with the composition from which anisotropic melt starts to form. A similar phenomenon was reported earlier by Kuhfuss and Jackson ${ }^{26}$ for POB/PET copolymers. Although the inherent viscosities and molecular weights of the liquid crystalline polymers were higher than those of the nonliquid crystalline ones, the complex viscosities of the former under high shear frequency were lower than those of the latter. ${ }^{26-30}$ mR-RES 86 , in spite of its high ${ }^{\circ} \eta_{\text {inh }}$ value, exhibits a lower complex viscosity. This may be due to the higher degree of molecular order or ease of molecular orientation in the melt of this composition than in the melts of isotropic compositions. Such a difference comes from the fact that molecular chain becomes stiffer and attains longer colinear chain segments as a higher level of POB moiety is incorporated. The experimental point for mR-RES 79 is located in the upper region from the main curve. This may be ascribed partially to its substantially higher molecular weight than others as reflected by its higher $\eta_{\text {inh }}$ value.

\section{CONCLUSIONS}

The following conclusions can be drawn from this investigation: Among the series of copolyesters synthesized, only those containing more than $65 \mathrm{~mol} \%$ of POB units are thermotropic, although those with 53 and $63 \mathrm{~mol} \%$ are able to form mesophases under shear. The compositions having either low or high amount of POB units are crystalline. But the compositions having a medium level of the POB moiety are amorphous as judged by DSC and X-ray analyses.

The torque of the reactor agitator encountered during polymerization is highly dependent on whether the composition is mesophase-forming or not. As opposed to isotropic compositions which exhibit steady ascent in torque with reaction time, the mesophase-forming ones show an initial steep increase followed by a slower, gentle increase. There is some evidence that the copolyesters containing high overall levels ( $>65 \mathrm{~mol} \%$ ) of the POB unit are heterogeneous in composition.

Acknowledgements. The authors are grateful to the Korea Science and Engineering Foundation and KOLON Inc. (Korea) for their support. 


\section{REFERENCES}

1. P. J. Flory, Adv. Polym. Sci., 59, 1 (1984).

2. S. P. Papkov, Adv. Polym. Sci., 59, 75 (1984).

3. M. G. Dobb and J. E. McIntyre, Adv. Polym. Sci., 60/61, 61 (1984).

4. J.-I. Jin, S. Antoun, C. Ober, and R. W. Lenz, Br. Polym. J., 12, 132 (1980).

5. C. K. Ober, J.-I. Jin, Q. Zhou, and R. W. Lenz, $A d v$. Polym. Sci., 59, 103 (1984).

6. J.-I. Jin and E-J. Choi, Macromolecules, 20, 934 (1987).

7. J.-I. Jin, J.-H. Chang, and H.-K. Shim, Macromolecules, in press.

8. J.-I. Jin, E-J. Choi, and S.-C. Ryu, J. Polym. Sci. Polym. Chem. Ed., 25, 241 (1987).

9. J.-I. Jin, H.-S. Choi, and C.-J. Yoon, J. Polym. Sci., Polym. Chem. Rd., in press.

10. J.-I. Jin, S.-H. Lee, and H.-J. Park, Polym. Bull., 20 19 (1988).

11. J.-I. Jin, J.-H. Chang, and B.-W. Jo, Polym. Bull., 20, 525 (1988).

12. W. R. Krigbaum, H. Hakemi, and R. Kotek, Macromolecules, 18, 965 (1985).

13. W. J. Jackson, Jr., Br. Polym. J., 12, 154 (1980).

14. B. Griffin and M. K. Cox, Br. Polym. J., 12, 147 (1980).

15. V. Percec and R. Yourd, Polym. Prepr., Am. Chem. Soc., Div., Polym. Chem., 29(1), 229 (1988).
16. W. Volksen, J. R. Lyerla, Jr., J. Economy, and B. J. Dawson, J. Polym. Sci., Polym. Chem. Ed., 21, 2249 (1983).

17. G. W. Calundann, U.S. Patent, 4,184,996 (1980).

18. G. A. Gutierrez and J. Blackwell, Macromolecules, 17, 2744 (1984).

19. W. J. Jackson, Jr. and J. C. Morris, U. S. Patent, 4,181,792 (1979).

20. J.-I. Jin, S.-H. Lee and H.-J. Park, Polym. Prepr., Am. Chem. Soc. Div., Polym. Chem., 28(1), 122 (1987).

21. M. Levine and S. Temin, J. Polym. Sci., 28, 179 (1958).

22. E. G. Joseph, G. L. Wilkes, and D. G. Baird, Polym. Eng. Sci., 25(7), 377 (1985).

23. R. Gilkey and J. R. Caldwell, J. Polym. Sci., II(5), 198 (1959).

24. K. F. Wissbrun, J. Rheol., 25(6), 619 (1981).

25. A. Bickel and M. T. Shaw, J. Rheol., 28(5), 647 (1984).

26. H. F. Kuhfuss and W. J. Jackson, Jr., J. Polym. Sci., Polym. Chem. Ed., 12, 2043 (1976).

27. R. E. Jerman and D. G. Baird, J. Rheol., 25(2), 275 (1981).

28. K. F. Wissburn, Br. Polym. J., 12, 163 (1980).

29. K. F. Wissburn and A. C. Griffin, J. Polym. Sci., Polym. Phys. Ed., 20, 1835 (1982).

30. A. D. Gotsis and D. G. Baird, J. Rheol., 29(5), 539 (1985). 